\title{
PEDOT:PSS/QCM-Based Multimodal Humidity and Pressure Sensor
}

\author{
Eric S. Muckley ${ }^{\mathrm{a}, \mathrm{b}^{*}}$, James Lynch ${ }^{\mathrm{a}}$, Rajeev Kumar ${ }^{\mathrm{a}, \mathrm{c}}$, Bobby Sumpter ${ }^{\mathrm{a}, \mathrm{c}}$, Ilia N. Ivanov ${ }^{\mathrm{a}^{*}}$ \\ a) Center for Nanophase Materials Sciences, Oak Ridge National Laboratory, Oak Ridge, TN 37831, USA \\ ${ }^{b)}$ Bredesen Center, University of Tennessee, Knoxville, TN 37996, USA \\ ${ }^{\text {c) }}$ Computer Science and Mathematics Division, Oak Ridge National Laboratory, Oak Ridge, TN 37831 , \\ USA
}

\begin{abstract}
A room temperature multimodal sensor composed of poly(3,4-ethylenedioxythiophene):polystyrene sulfonate (PEDOT:PSS) deposited on an AT-cut quartz crystal microbalance (QCM) crystal was fabricated. The sensor's nonlinear motional resistance and frequency responses are deconvoluted using a feedforward backpropagation neural network (FBN), which allows a single sensor to function simultaneously as a relative humidity (RH) sensor and a pressure sensor using only two electrodes. We demonstrate that the predictive ability of the sensor is highly influenced by the data used to train the FBN. When training sets are tailored to resemble the operating conditions of the sensor, the sensor achieves an average resolution of $<4 \% \mathrm{RH}$ from $0-100 \% \mathrm{RH}$, even after $\mathrm{H}_{2} \mathrm{O}$ saturation occurs on the surface. Our results indicate that FBNs show strong promise for improving the resolution of low cost gas sensors and for expanding the range of environmental conditions in which a given sensor can operate.
\end{abstract}

\section{Graphical abstract}

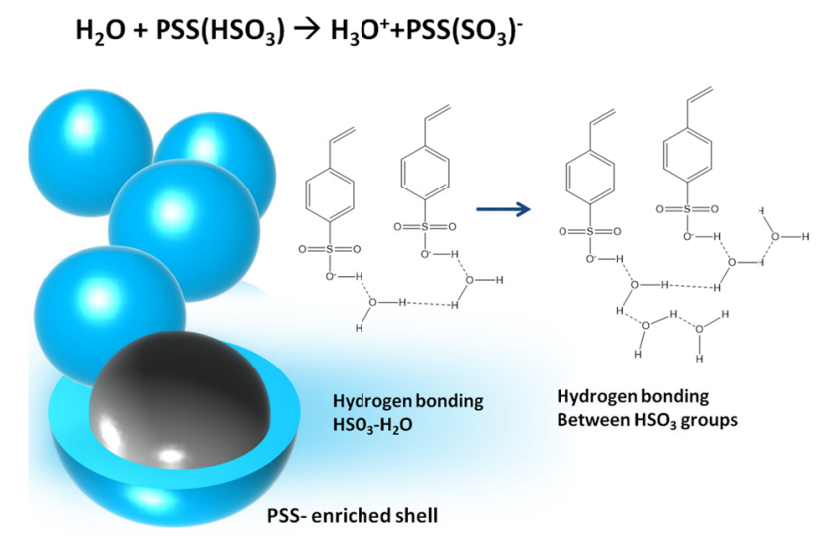

Keywords: PEDOT:PSS, QCM, QCM response modeling, neural network, humidity, pressure

*Corresponding authors: Ivanov, Ilia N. ivanovin@ornl.gov, Muckley, Eric S. muckleyes@ornl.gov

\section{Introduction}

Growing interest in personal wearable sensors drives research activity toward the development of versatile, multifunctional polymer sensors for environmental and bionic sensing ${ }^{1-3}$. An ideal sensor material has high sensing selectivity for a particular environmental parameter like humidity, temperature or pressure. Alternatively, other environmental parameters are measured by auxiliary sensors and the response of the main sensor is corrected. Commercial relative 
humidity (RH) sensors based on polymer films are sensitive to pressure and temperature, requiring correction procedures ${ }^{4-6}$. Several optical sensors capable of simultaneous measurement of ambient pressure and $\mathrm{RH}$ have been demonstrated, but with limited application for high humidity environments $(\mathrm{RH}>70 \%)^{7,8}$. The quartz crystal microbalance (QCM) is a commonly used sensor platform for vacuum deposition systems and gas sensing ${ }^{9-12}$ but its use is limited when pressure and analyte concentration are changing simultaneously, and most experiments report results at isobaric and iso-humid conditions to avoid complicated correction procedures $^{4,13,14}$.

Recent efforts in environmental sensing have shown that application of a feedforward backpropagation neural network (FBN), a machine-learning technique optimized for pattern recognition and classification problems ${ }^{15,16}$, enables deconvolution of sensor response in challenging gas environments ${ }^{17-20}$. The benefit of FBNs is accentuated for systems in which a limited number of sensors can measure a broad range of environmental parameters.

Here, we demonstrate a single multimodal pressure and RH sensor based on the polymer mixture poly(3,4-ethylenedioxythiophene):polystyrene sulfonate (PEDOT:PSS) deposited on a QCM crystal. PEDOT:PSS has been widely-studied for environmental sensing applications because of its low-cost synthesis and processing ${ }^{21,22}$ and its efficient response to humidity ${ }^{23,24}$, pressure $^{25}$, temperature ${ }^{26}$, and light ${ }^{27}$.

Traditional PEDOT:PSS RH sensor response is based on electronic conductivity of the film. As depicted in Figure 1, the PEDOT:PSS film consists of PEDOT-rich domains embedded in a PSS matrix. Diffusion of $\mathrm{H}_{2} \mathrm{O}$ into PEDOT:PSS films causes film swelling due to protonation of $\mathrm{SO}_{3} \mathrm{H}^{+}$groups at the PEDOT-PSS interface, resulting in the formation of $\mathrm{H}_{3} \mathrm{O}^{+} \mathrm{PSS}\left(\mathrm{SO}_{3}\right)^{-}$. Swelling of the film increases the distance between adjacent PEDOT domains, leading to a decrease in charge carrier mobility ${ }^{24,28,29}$. Hydrogen bonding between adsorbed $\mathrm{H}_{2} \mathrm{O}$ and vaporphase $\mathrm{H}_{2} \mathrm{O}$ accelerates $\mathrm{H}_{2} \mathrm{O}$ sorption and leads to further film swelling. $\mathrm{H}_{2} \mathrm{O}$ sorption disrupts hydrogen bonding between PSS molecules, leading to changes in viscosity and shear modulus of the polymer matrix (see Supporting Information). The electrical resistivity of PEDOT:PSS increases linearly as $\mathrm{RH}$ increases $(\mathrm{RH}<60 \%)^{28}$. At higher values of $\mathrm{RH}(>60 \%)$, a water meniscus is formed on the PEDOT:PSS surface resulting in highly non-linear sensor response over a broad range of humidities ${ }^{1,3,30}$. Analysis of the nonlinear QCM sensor response is achieved here by application of an FBN, which does not rely on the electronic response of PEDOT:PSS. 


\section{$\mathrm{H}_{2} \mathrm{O}+\mathrm{PSS}\left(\mathrm{HSO}_{3}\right) \rightarrow \mathrm{H}_{3} \mathrm{O}^{+}+\mathrm{PSS}\left(\mathrm{SO}_{3}\right)^{-}$}
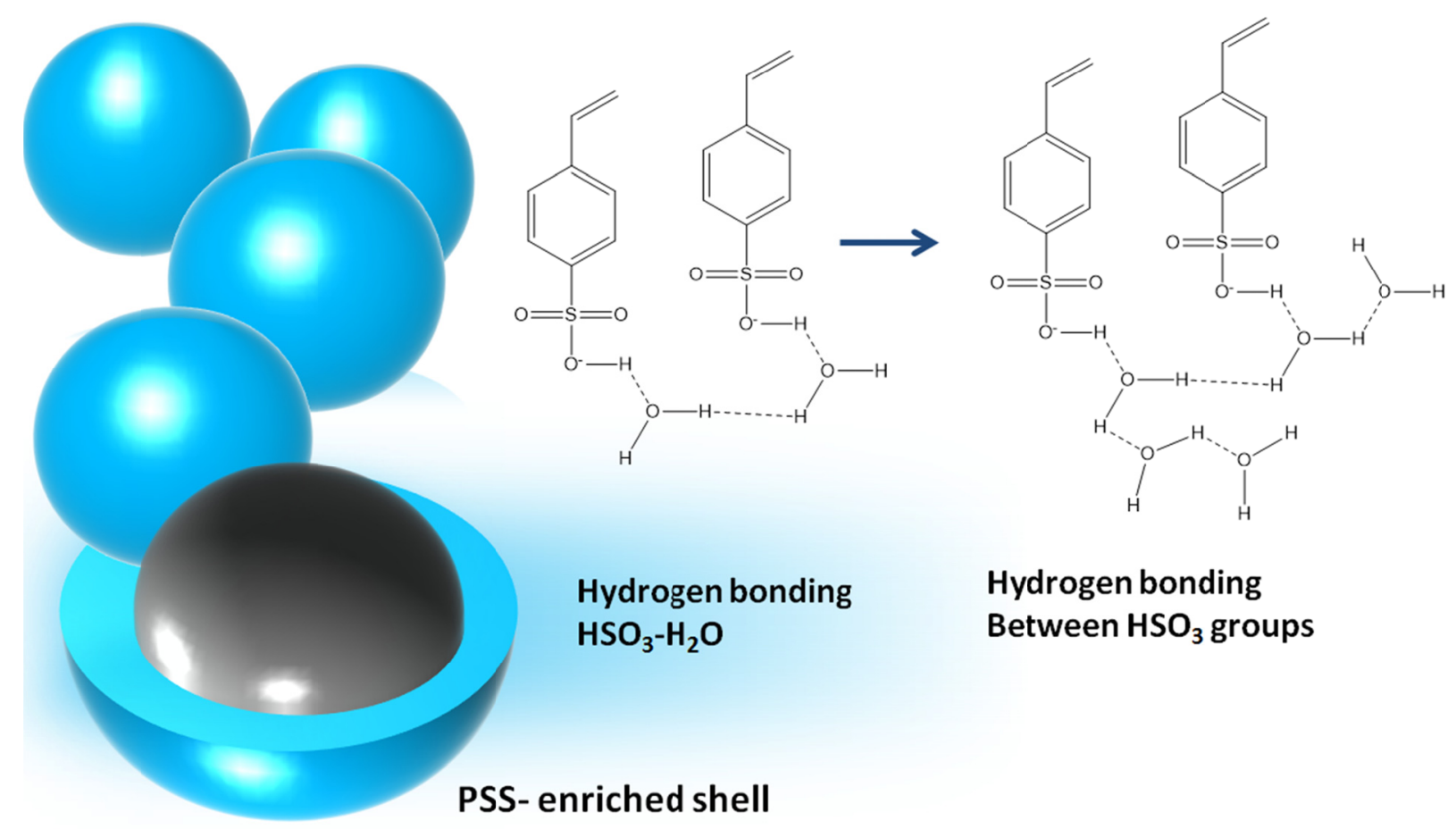

Hydrogen bonding

Between $\mathrm{HSO}_{3}$ groups

Figure 1. Schematic of hydrogen bonding between PSS and water and PSS-PSS molecules within the PEDOT:PSS film. Diffusion of $\mathrm{H}_{2} \mathrm{O}$ into the film causes swelling due to protonation

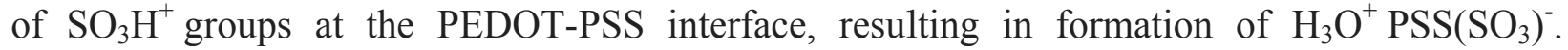
Polymer swelling and hydrogen bonding between $\mathrm{H}_{2} \mathrm{O}$ adsorbates and vapor result in modification of the viscoelastic properties of the film.

For a QCM coated with a thin rigid film that undergoes uniform mass loading, the change in oscillation frequency of the crystal $(\Delta f)$ is described by the Sauerbrey equation

$$
\Delta f=-\frac{2 f_{0}^{2}}{A \sqrt{\rho_{q} \mu_{q}}} \Delta m
$$

where $\Delta m$ is the mass change of the film/crystal, $f_{0}$ is the resonant frequency of the crystal, and $A, \rho_{q}$, and $\mu_{q}$ are the area, mass density, and shear modulus of the crystal ${ }^{31}$. However, validity of the Sauerbrey relation is limited for PEDOT:PSS films under dynamic RH and pressure conditions because viscoelastic properties of the film change as a result of sorption-induced polymer swelling and pressure-induced film compression ${ }^{25}$. Understanding shifts in the resonance frequency of an oscillating crystal in contact with viscoelastic polymer film requires detailed models that include effects of viscosity, shear modulus of the polymer, and other phenomena such as hydrodynamic screening and slip at different interfaces. Complimentary information about the viscoelastic properties of the film can be obtained by analysis of an equivalent circuit model for the QCM coated with the film. One such model is the Butterworthvan Dyke (BvD) equivalent circuit, which consists of a static capacitive branch representing the QCM crystal in parallel with an acoustic branch that contains motional inductive, capacitive, and resistive components ${ }^{32}$. Muramatsu et al. have shown how to relate different elements of the 
equivalent circuit model to mechanical motion, leading to correspondence between inductance and mass, capacitance and compliance, and resistance and friction via the electromechanical coupling factor $\mathrm{k}^{33}$.

The motional resistance $\left(R_{m}\right)$ is related to energy damping of the resonator and contains contributions from internal frictions in the crystal, mechanical losses in the mounting system, and the viscosity of the film deposited on the crystal ${ }^{34}$. For a two fluid model of a crystal oscillator in contact with a liquid, one can derive the contribution to $R_{m}$ that occurs as a result of the film/liquid interaction (see the Supporting Information for the details). Since internal friction in the crystal and mechanical losses in the mounting system are independent of $\mathrm{RH}$, we can assume that all environment-induced changes in $R_{m}$ occur as a result of $\mathrm{H}_{2} \mathrm{O}$-induced changes in the viscoelastic properties of the film. Thus, by continuous monitoring of $R_{m}$ along with $\Delta f$, it is possible to observe viscoelastic changes in the PEDOT:PSS film as a function of vapor pressure of $\mathrm{H}_{2} \mathrm{O}$. By measuring both $R_{m}$ and $\Delta f$ changes in PEDOT:PSS on a QCM, we demonstrate that utilization of a pattern recognition tool such as the FBN allows for deconvolution of the pressure and RH response, enabling the single sensor to perform continuous measurements of both RH and pressure.

\section{Experiment}

For fabrication of the sensor, a PEDOT:PSS (ratio of 1:6) solution (Aldrich) was spin coated at $2000 \mathrm{rpm}$ for 90 seconds to achieve a $50 \mathrm{~nm}$ thick film on a gold-plated, AT-cut $5 \mathrm{MHz}$ QCM crystal. Prior to testing, the sensor was placed inside a vacuum chamber at pressure $10^{-6}$ Torr vacuum for 4 hours. A LabVIEW program was used to control pressure and $\mathrm{RH}$ inside the vacuum chamber by adjusting the flow of argon and $\mathrm{H}_{2} \mathrm{O}$ vapor with two mass flow controllers. The $\mathrm{RH}$ was regulated by admitting vapor from a sealed $\mathrm{H}_{2} \mathrm{O}$ vessel into the testing chamber using the flow controller. The QCM frequency and motional resistance were measured using an SRS QCM 200 and recorded with a LabVIEW program. All measurements were performed at room temperature. Since $\mathrm{RH}$ depends only on $\mathrm{H}_{2} \mathrm{O}$ vapor pressure and temperature, changing argon pressure at constant temperature did not affect $\mathrm{RH}$ levels inside the testing chamber.

For configuration of the FBN, a feed-forward network was trained using the Neural Net Fitting application in MATLAB. QCM frequency change and motional resistance were used as the two FBN inputs $\left(I_{i}\right)$ fed into 1 hidden layer with 5 neurons, where RH and pressure are used as outputs, as shown in Figure 2a. The standard sigmoid logistic function $T\left(S_{i}\right)$ was used as a transfer function. To investigate the effect of training sets on the predictive ability of the PEDOT:PSS sensor we constructed two training sets: (1) $R_{m}$ and frequency response to pulsed Ar and $\mathrm{H}_{2} \mathrm{O}$ pressures (Figure $2 \mathrm{~b}$ top) and (2) $R_{m}$ and frequency response to continuously varying $\mathrm{Ar}$ and $\mathrm{H}_{2} \mathrm{O}$ pressures (Figure $2 \mathrm{~b}$ bottom). The predictive ability of the sensor was then tested in both continuously varying and pulsed pressure and $\mathrm{RH}$ conditions using both types of training sets. About 13,000 sets of training data and 1,000 iterations of the weights $\left(W_{i}\right)$ were used for FBN training, which required about 3 minutes. $70 \%$ of the data was used for training, $15 \%$ for testing, and $15 \%$ for validation of the network. FBN performance changed by less than $0.5 \%$ when the network was re-trained using the same training configuration and data, so a bootstrap statistical approach was not used. To optimize the number of neurons in the hidden layer, identical experiments were repeated for networks with $2,3,4,5,6,8,10,12$, and 15 hidden 
neurons. Since the network with 5 hidden neurons achieved the lowest testing/training errors, we report only results from that network.

The sensor response used for FBN training in continuously varying conditions is shown in Figure 2c. The sensor $R_{m}$ and frequency changes are measured at continuously changing pressures of both $\mathrm{Ar}$ gas and $\mathrm{H}_{2} \mathrm{O}$ vapor. While the QCM frequency (see inset in Figure 2c) is independent of Ar pressure which indicates that no Ar adsorption takes place on the film, it decreases exponentially with increasing $\mathrm{H}_{2} \mathrm{O}$ pressure. The exponential behavior of the frequency change is related to mass loading by the Sauerbrey equation and resembles the type III BET isotherm, which is characteristic of adsorption by a species which bonds more readily to other adsorbate molecules than to the adsorbent material ${ }^{35}$. This suggests that after the initial adsorption of $\mathrm{H}_{2} \mathrm{O}$ on the PEDOT:PSS surface, hydrogen bonding between adsorbed $\mathrm{H}_{2} \mathrm{O}$ and gas phase $\mathrm{H}_{2} \mathrm{O}$ dominates the adsorption process. This effect presents a significant problem for traditional PEDOT:PSS-based resistive sensors because it allows for heterogeneous adsorption in which multiple layers of $\mathrm{H}_{2} \mathrm{O}$ form puddles on the film surface even before complete monolayer formation has occurred. Our sensor avoids this problem because it does not rely on the electronic response of PEDOT:PSS.

The FBN was trained using gas pulses with discrete pressure and RH values for construction of a matrix of over 100,000 data points. A sample representation of this training set is shown in Figure 2 d. $R_{m}$ exhibits a local maximum near $60 \% \mathrm{RH}$, which is consistent with the data shown in Figure 2c. It is important to note that the contour profiles of the two figures are qualitatively different, which indicates some orthogonality between the two sensor inputs. Since the sensor is limited to two inputs, this orthogonality is required for enabling the measurement of two distinct environmental conditions. 

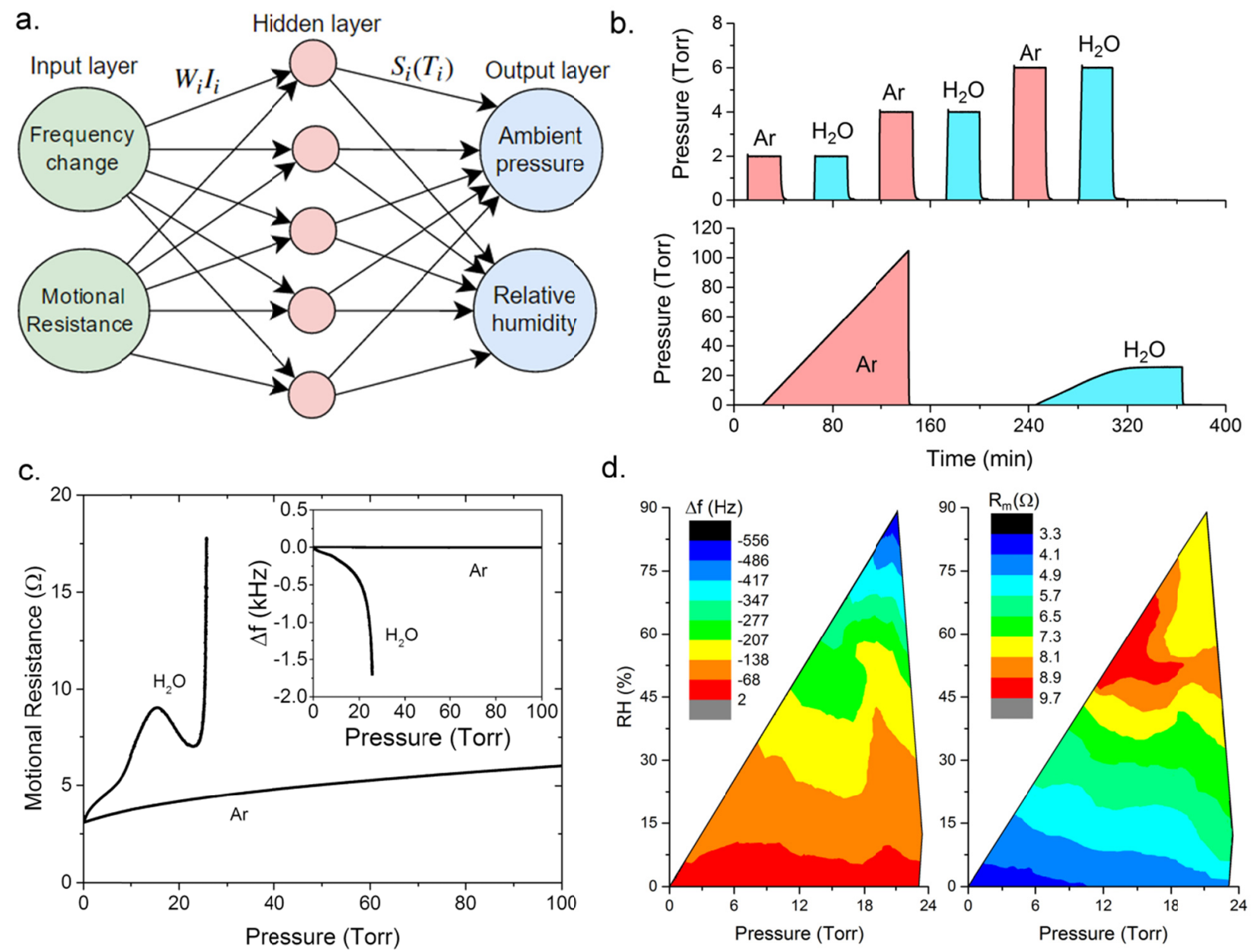

Figure 2. a. The FBN uses QCM frequency change and motional resistance as inputs $I_{i}$, the logistic function $T\left(S_{i}\right)$ as an activation function, and $\mathrm{RH}$ and ambient pressure as outputs. $\mathrm{b}$. Examples of training data sets in which $\mathrm{RH}$ and pressure conditions are changed by discrete values using pulses (top) and continuously varied (bottom). c. An example of FBN training data generated by continuously varying Ar pressure and RH. d. An example of FBN training data generated by a matrix of pulses with discrete $\mathrm{Ar}$ and $\mathrm{H}_{2} \mathrm{O}$ ratios.

\section{Results and Discussion}

The motional resistance shown in Figure $2 \mathrm{c}$ is a consequence of resonator damping by the viscoelastic PEDOT:PSS film. A clear distinction in response to pressure is seen while comparing cases of $\mathrm{Ar}$ and $\mathrm{H}_{2} \mathrm{O}$. When the sensor is exposed to $\mathrm{Ar}, R_{m}$ exhibits a monotonic increase with increases in pressure. This is expected for a mechanical resonator subject to increasing pressure of an inert gas since the gas exerts a drag-like damping force on the oscillator. In contrast, $R_{m}$ exhibits non-monotonic response with increases in $\mathrm{H}_{2} \mathrm{O}$ pressure. In order to understand the origin of the non-monotonic response, we have used a viscoelastic description based on the assumption that a hydroscopic polymer film undergoing $\mathrm{H}_{2} \mathrm{O}$ sorption can be described by a two-fluid model in which the viscoelastic properties of the film change as a result of the interaction between the polymer and $\mathrm{H}_{2} \mathrm{O}^{36}$. Using the two fluid model in the thin film limit (see Supporting Information for details), the $\Delta f$ and $R_{m}$ responses are modeled in 
Figure $3 \mathrm{a}$ and $3 \mathrm{~b}$. In particular, we have modeled the responses by varying the film thickness $(\mathrm{d})$ and viscosity of water $(\eta)$ as a function of $\mathrm{H}_{2} \mathrm{O}$ vapor pressure $(p)$. Dependence of the film thickness on the pressure is derived (Eq. S-9 in the Supporting Information) by assuming that water molecules adsorb on the film and the adsorption strength is characterized via the Hamaker constant $(A)$. Empirical relation for pressure dependence of viscosity of water $(\eta)$ was used so that $\eta=\eta_{0} \exp \left(\eta_{1} p+\eta_{2}\right)$, where the parameters $\eta 0$ is the viscosity of water at ambient conditions, and $\eta 0 \exp (\eta 2)$ is the viscosity of water at zero pressure and $\eta 1$ is the parameter which captures the pressure dependence of the viscosity. The colored segments in Figures $3 \mathrm{a}$ and $3 \mathrm{~b}$ correspond to different values of $A, \eta_{1}$ and $\eta_{2}$. Physically, the changes in $A$ and $\eta_{1}$ represent step-changes in the film thickness and viscosity of the water, respectively. These changes in film thickness $\left(d-d_{o}\right.$, so that $d_{o}$ is the initial film thickness) and viscosity $(\eta)$ are shown in Figure $3 c$ and $3 \mathrm{~d}$, respectively, with the same color scheme. From Figures $3 \mathrm{c}$ and $3 \mathrm{~d}$, we find that both the film thickness and the viscosity increase with an increase in the pressure, and estimates for the increase in their values based on the modeling of $\Delta f$ and $R_{m}$ data are in agreement with each other. The increase in the film thickness is expected due to the adsorption of water molecules on the film and it is also reasonable to assume that the adsorption energy characterized by $A$ may vary due to viscoelastic changes in the film. Similarly, an increase in the viscosity of water with an increase in pressure is also expected due to slowing down of the dynamics of water molecules. However, the non-monotonic behavior of $R_{m}$ hints at step decrease in film thickness and the viscosity as shown in Figure $3 \mathrm{c}$ and $3 \mathrm{~d}$, respectively. Origin of these nanoscopic changes is beyond the scope of this work and we speculate this may arise due to diffusion of water inside the film. Furthermore, the decrease in $R_{m}$ with an increase in $p$ highlights the breakdown of proportionality between $R_{m}$ and $\Delta f$, strictly valid in the limit of very small $\Delta f$.

The complex behavior of traditional resistive PEDOT:PSS-based RH sensors at relative humidity above $60 \%$ typically restricts RH sensor operation to either low, mid-range, or high RH regimes $^{24,28-30}$. The unique feature of the $\mathrm{RH}$ sensor reported here is that it relies on two inputs. While the $R_{m}$ response of the sensor exhibits complex behavior, the frequency response remains monotonic, which allows for greater predictability over a wide range of conditions. This permits the sensor to measure humidity from dry vacuum environments ( $\mathrm{RH} \sim 0 \%$, pressure $\sim 10^{-6}$ Torr) to those with $\mathrm{RH} \sim 100 \%$. Due to their nonlinearity, deconvolution of the pressure and $\mathrm{RH}$ response over the entire $0-100 \% \mathrm{RH}$ range is not possible without the use of a powerful tool like the FBN. 

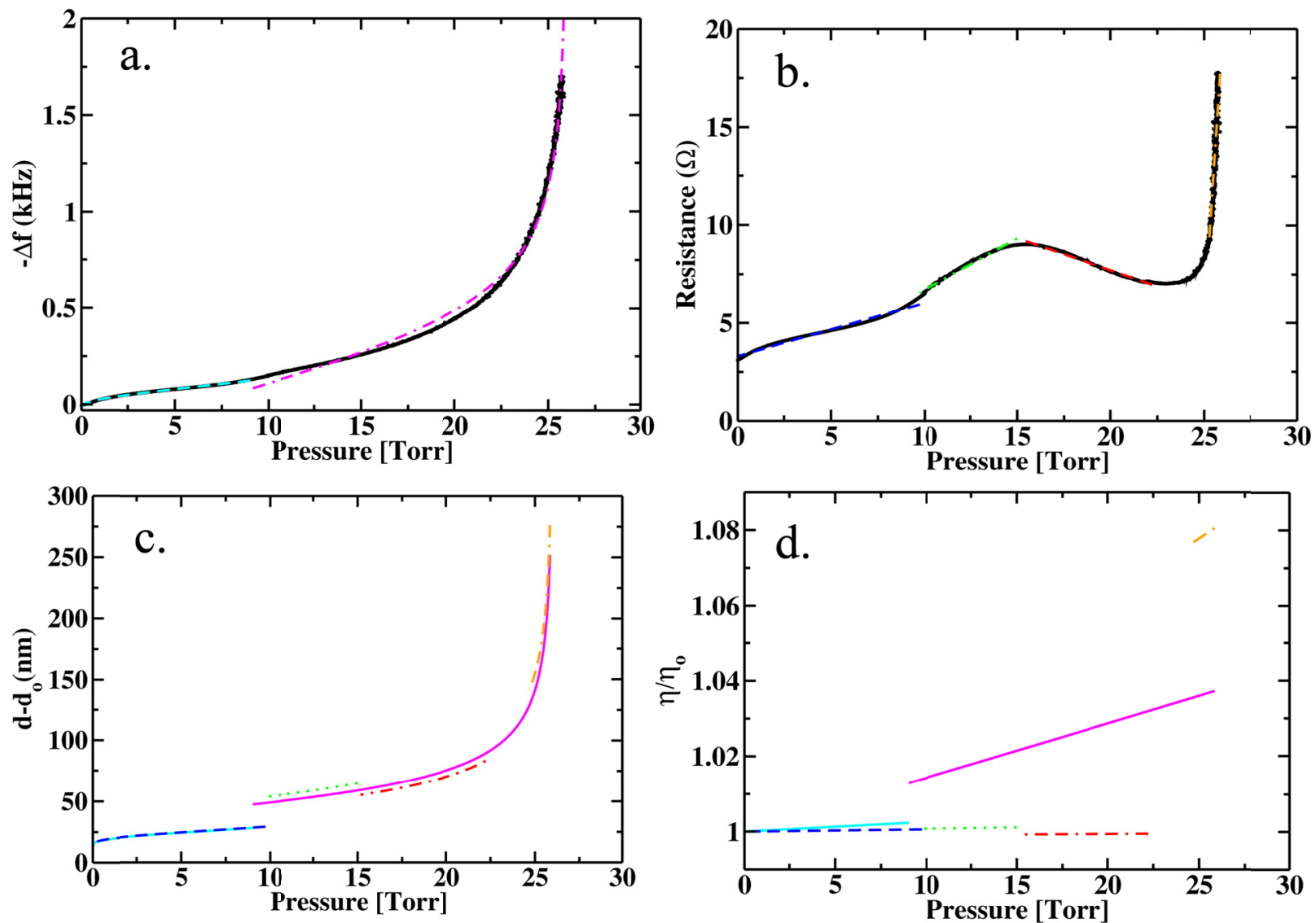

Figure 3. Modeling of (a) $\Delta f$ and (b) $R_{m}$ responses in the presence of $\mathrm{H}_{2} \mathrm{O}$ using the thin film limit of a two fluid model. Experimental data is shown in black along with predictions of the model (colored segments) corresponding to different values of the Hamaker constant and viscosity ( $\eta$ )-pressure relations used to fit the data. Corresponding changes in film thickness (c) and viscosity (d) are determined based on the modeling of $\Delta f$ and $R_{m}$. The color scheme is kept the same in each figure for clarity. 

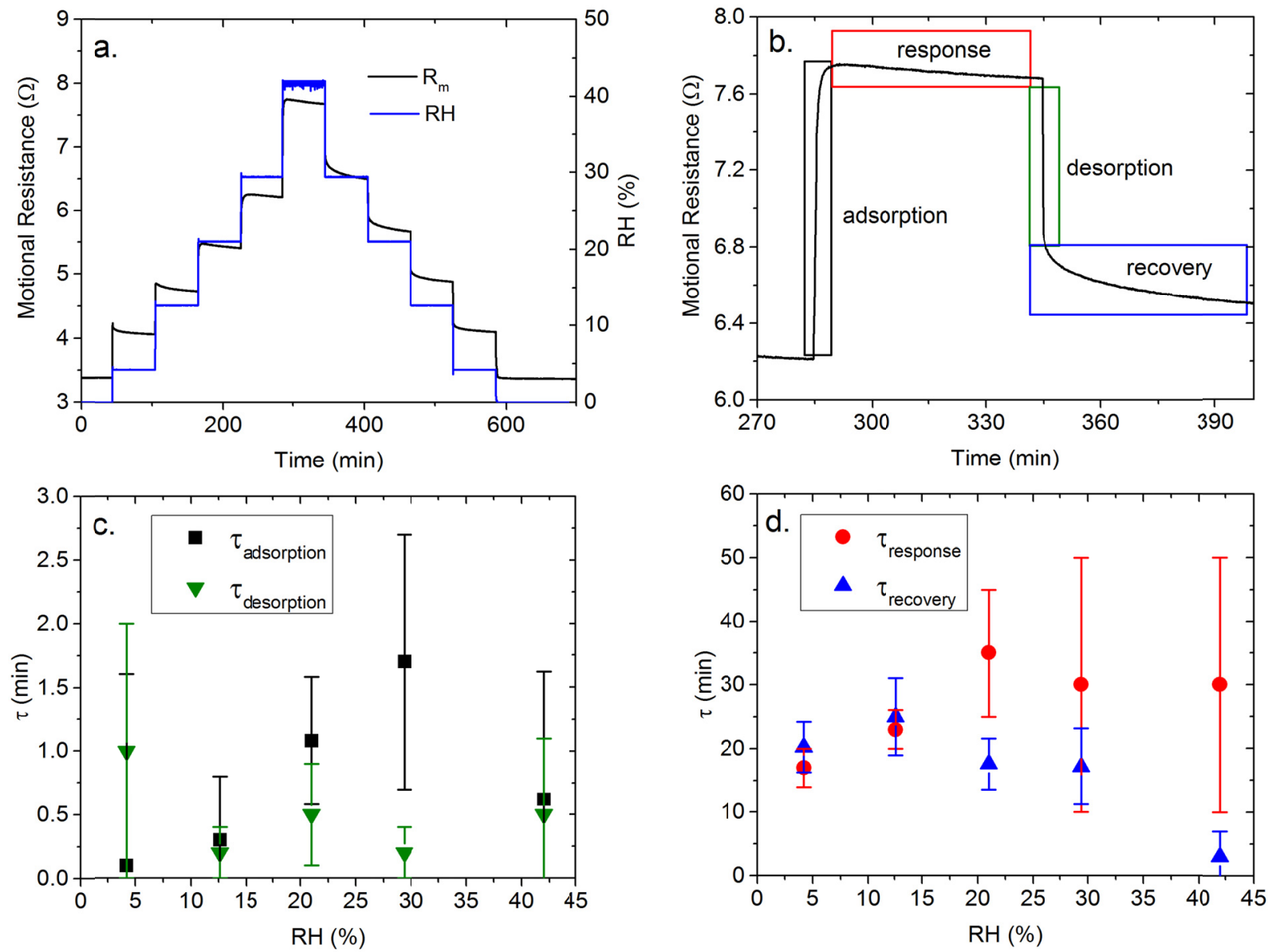

Figure 4. a. At low $\mathrm{RH}$, the $R_{m}$ response to changing humidity levels exhibits stability, short response time, and reversibility. $b$. The sensor exhibits behavior which can be represented by four distinct processes. For clarity, they are labeled adsorption, response, desorption, and recovery. Response at $40 \% \mathrm{RH}$ is shown here. c. The rapid ( $\tau \sim 1 \mathrm{~min})$ adsorption and desorption processes exhibit similar time constants, and $\mathrm{d}$. the longer $(\tau \sim 25 \mathrm{~min})$ response and recovery processes exhibit similar time constants. This suggests that the sensing behavior is fully reversible.

In order to characterize the sensor stability, response time, and reversibility, the $R_{m}$ response to a sequence of RH steps is shown in Figure 4a. The RH conditions shown in Figure 4a take place in the low pressure regime where the $R_{m}$ response remains monotonic (see Figure $2 \mathrm{c}$ ). The sensor response exhibits less than $4 \%$ drift over the 60 minute long RH steps, which is regarded as very stable for a RH sensor ${ }^{2,37}$. The sensor requires about 40 seconds to achieve full response to a change from $0 \% \mathrm{RH}$ to $40 \% \mathrm{RH}$. This is on the same order of magnitude as the response time of a leading manufacturer of RH sensors, which reports 10 seconds to achieve $90 \%$ of full response to an increase of $11 \%$ to $93 \% \mathrm{RH}^{6}$. Changes in QCM motional resistance during the $\mathrm{H}_{2} \mathrm{O}$ adsorption-desorption process are shown in Figure $4 \mathrm{~b}$. The kinetics of reversible water adsorption resemble exponential behavior of the form $R_{m}=R_{m 0} \exp (-\mathrm{t} / \tau)$, where each process can be characterized by its time constant $(\tau)$. For clarity, the four distinct processes have been labeled adsorption, response, desorption, and recovery in Figure 4b. During introduction of vapor to the 
testing chamber, the adsorption process occurs while $\mathrm{d} R_{m} / \mathrm{dt}>0$, and the response regime occurs when $\mathrm{d} R_{m} / \mathrm{dt} \leq 0$. During gas/vapor removal, desorption occurs while $\mathrm{d}^{2} R_{m} / \mathrm{dt}^{2} \approx 0$ and the recovery cycle begins when $\mathrm{d}^{2} R_{m} / \mathrm{dt}^{2}>0$. Since the four kinetic processes are divided into nearly vertical (average $\left|\mathrm{d} R_{m} / \mathrm{dt}\right|>>1$ ) and horizontal (average $\left|\mathrm{d} R_{m} / \mathrm{dt}\right|<<1$ ) segments, the fitting parameter $\left|R_{m 0}\right|$ is very large $\left(\left|R_{m 0}\right|>10^{10}\right)$ during adsorption, desorption, and recovery regimes. Because of the large uncertainties associated with $R_{m 0}$, these values are not discussed in detail here.

The rapid increase in $R_{m}$ during the adsorption regime is a consequence of $\mathrm{H}_{2} \mathrm{O}$ surface layer formation and polymer swelling near the film surface, while the rapid decrease in $R_{m}$ during the desorption regime corresponds to the removal of surface $\mathrm{H}_{2} \mathrm{O}$ and shrinkage of the film as revealed in the modeling of the $R_{m}$ response in Figure 3. The time constant $\tau$ of each process is extracted as a function of RH and shown in Figure 4c for adsorption and desorption and Figure $4 \mathrm{~d}$ for response and recovery. As seen in Figure $4 \mathrm{c}, \tau \sim 1 \mathrm{~min}$ for the adsorption and desorption processes, which suggests that adsorption and desorption represent the forward and reverse kinetics of the same rapid process. Similarly, $\tau \sim 25 \mathrm{~min}$ for the response and recovery processes (Figure 4d), which suggests that response and recovery represent the forward and reverse kinetics of the same relatively slow process. The similarity in time constants suggests that the $\mathrm{H}_{2} \mathrm{O}$ sorption-desorption process is reversible on the sensor. The mechanisms behind the kinetics for each process will be investigated in future studies.
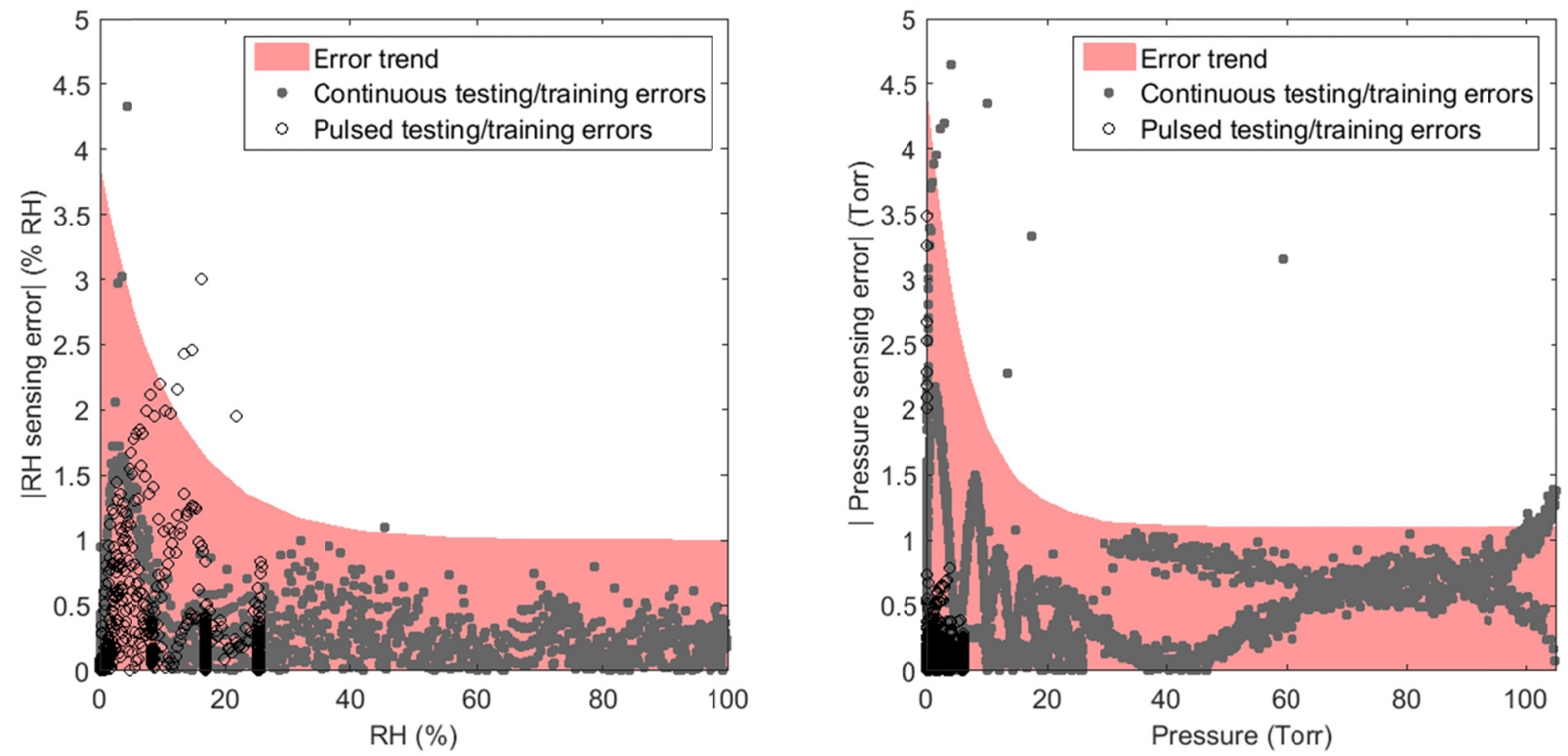

Figure 5. FBN-assisted sensor error in measuring RH (left) and pressure (right) when the sensor is tested in conditions similar to those used during training (i.e. both training and testing are conducted under either continuously varying or pulsed conditions).

To investigate the importance of the specific data set used for FBN training, the predictive ability of the sensor was trained and tested under both continuously varying conditions and pulsed conditions. The sensor exhibited average errors of 10-30\% (data not presented) when trained under conditions different than those in which it was tested. This is a consequence of overtraining, a common problem with FBNs in which the network makes predictions based on a 
training data set which either exceeds the optimal training size or includes data which is irrelevant to the desired output, such as noise or artifacts in the data. In this case, the FBN trained with pulsed data is over-trained to recognize only specific discrete values of $\mathrm{RH}$ and pressure. Similarly, networks trained under continuously varying conditions are unconditioned to provide accurate measurements during abrupt gas pulses.

When the FBN is tested under conditions similar to those in which it was trained, the accuracy of the sensor increases by an order of magnitude or more, as shown in Figure 5 for RH sensing (left) and pressure sensing (right). Appropriate training enables the sensor to achieve an error of less than $4 \% \mathrm{RH}$ from $0-100 \% \mathrm{RH}$ and less than 5 Torr from $0-100$ Torr. The error here is defined as the difference between the output of the FBN-assisted sensor and measurements made by a commercial pressure sensor. The errors for each of the 1,950 sensor testing experiments are depicted by solid gray circles for continuous training/testing and open black circles for pulsed training/testing. For both $\mathrm{RH}$ and pressure, the magnitude of the error follows a roughly exponential trend (shown in red). Above $\sim 40 \% \mathrm{RH}$ a relatively constant error of around $1 \%$ is maintained. At low RH and pressure, the sensor response to $\mathrm{H}_{2} \mathrm{O}$ and $\mathrm{Ar}$ is similar (as shown in Figure 2c), which makes it difficult to distinguish between $\mathrm{RH}$ and pressure at low pressure $(<10$ Torr) conditions. This effect results in the higher errors at low RH and pressure. It should be noted that for industrial applications, accurate pressure measurement over a wide range of pressures often requires the use of multiple sensors which operate in different pressure regimes. While the sensor reported here is optimized for mid-range (20-100 Torr) pressures it is possible to utilize multiple training sets tailored toward different environmental regimes. For example, training the sensor with data from atmospheric conditions would enable $\mathrm{RH}$ and pressure sensing with a similar $(4 \%)$ resolution at atmospheric conditions. Since the focus of this study is to investigate the application of FBNs for deconvoluting nonlinear sensor response, we limit our discussion to the pressure regime in which a nonlinear sensor behavior exists $(<100$ Torr).

It is important to discuss the behavior of the continuous testing errors in the right side of Figure 5. The errors at pressures $<20$ Torr exhibit oscillatory behavior around 0 as the FBN predictions approach convergence near 30 Torr. At this pressure, a new trend appears near $1 \%$ error. This set of points can be explained by the configuration of the continuously varying training conditions shown in the bottom of Figure $2 \mathrm{~b}$. Since the Ar pressure ramp increases up to 100 Torr and then falls back toward 0 Torr, the sensor is trained and tested twice at each pressure: once while the pressure is gradually increasing, and once while it abruptly decreases. This causes the appearance of two distinct error sets in the right side of Figure 5 after 30 Torr. The set of higher errors corresponds to evacuation of the chamber and the abrupt drop in pressure, while the lower error values correspond to the continuously increasing Ar pressure. The continuously varying data used to train the sensor diverges at $\mathrm{H}_{2} \mathrm{O}$ pressures $>23$ Torr (as shown in Figure 2c), which adds nonlinearity to the training data, resulting in lower accuracy for predictions of high pressures. Since this effect is a consequence of over-training at high $\mathrm{RH}$, it would not be present for a single sensor which was trained solely for the purpose of predicting pressure. This suggests that the sensor accuracy is necessarily limited by its multi-modal capability, indicating that a balance between functionality and accuracy must be chosen for the specific application in which the sensor is deployed. 
The significant result reported in Figure 5 is that the $\mathrm{RH}$ sensor trained by the appropriate data exhibits a resolution of less than $4 \%$ from $0-100 \%$ RH. For comparison, Sreenivasan et al. report $10 \%$ error from a wide-range optical $\mathrm{RH}$ sensor which is operational in $20 \%-70 \% \mathrm{RH}$ conditions $^{38}$. Furthermore, Kuş and Okur report that at RH $>80 \%$, PEDOT:PSS ceases to function as a $\mathrm{RH}$ sensor because of the formation of a $\mathrm{H}_{2} \mathrm{O}$ meniscus on the film surface ${ }^{30}$. Our sensor is not limited to $\mathrm{RH}<85 \%$ because the frequency response remains monotonic even as $\mathrm{RH}$ approaches $100 \%$. One of the most significant advantages of the FBN is its ability to extract information from the sensor response even in post-saturation/nonlinear regimes which typically excluded. Since our sensor relies on both $\Delta f$ and $R_{m}$, the FBN can interpret data even after one of the inputs diverges due to high $\mathrm{RH}$ conditions, which enables measurement of RH in the entire range from $0-100 \% \mathrm{RH}$.

Training with data sets which are similar in nature (i.e., ramped instead of pulsed) to the actual conditions in which the sensor is deployed allow for significant improvements in FBN accuracy. By training the FBN to recognize different pressure/RH regimes, the sensor can be effectively tuned to operate accurately under a specific set of conditions. This provides a flexible platform for multimodal sensors that can be trained for operation under arbitrary humidity conditions. Future optimization of the FBN will include testing its predictive ability when gases of mixed composition are exposed to the sensor. Although the present experiment was performed at room temperature, it is important to evaluate the sensor performance under conditions with temperature variability as well. Subsequent investigations will study the importance of temperature on the response and predictive ability of the sensor. It is expected that if temperature is incorporated into the training set of the FBN as a third input, it will be possible for sensor measurements to accommodate thermal drift issues.

\section{Conclusion}

We have demonstrated the possibility of using an FBN-assisted PEDOT:PSS/QCM-based sensor to make simultaneous measurements of both ambient pressure and RH. The multi-input configuration of the sensor allows for accurate $(<4 \%$ error) $\mathrm{RH}$ sensing over a wide range of $\mathrm{RH}$ conditions $(0-100 \%)$, even after $\mathrm{H}_{2} \mathrm{O}$ meniscus formation occurs on the film surface. We have shown that the predictive ability of the sensor is highly influenced by the quality of the training data set. It is expected that with further optimization of the FBN training set, the addition of temperature dependence studies, and utilization of a more powerful computational tool such as the feedforward deep network or generalized regression network, future investigations can achieve higher prediction accuracies under a wider range of environmental conditions.

\section{Acknowledgement}

This research was conducted at the Center for Nanophase Materials Sciences, which is a DOE Office of Science User Facility. James Lynch acknowledges support from the Science Undergraduate Laboratory Internships (SULI) program of the DOE. 


\section{References}

[1] H. Farahani, R. Wagiran, and M. N. Hamidon. "Humidity sensors principle, mechanism, and fabrication technologies: A comprehensive review," Sensors 14, 7881-7939 (2014). DOI: $10.3390 / \mathrm{s} 140507881$

[2] Z. Chen and C. Lu. "Humidity sensors: a review of materials and mechanisms," Sensor letters 3, 274-295 (2005). DOI: 10.1166/sl.2005.045

[3] O. K. Varghese and C. A. Grimes. "Metal oxide nanoarchitectures for environmental sensing," Journal of Nanoscience and Nanotechnology 3, 277-293 (2003). DOI: 10.1166/jnn.2003.158

[4] N. Han, Y. Tian, X. Wu, and Y. Chen. "Improving humidity selectivity in formaldehyde gas sensing by a two-sensor array made of Ga-doped ZnO," Sensors and Actuators B: Chemical 138, 228-235 (2009). DOI: 10.1016/j.snb.2009.01.054

[5] H. Shurmer, J. Gardner, and H. Chan. "The application of discrimination techniques to alcohols and tobaccos using tin-oxide sensors," Sensors and Actuators 18, 361-371 (1989). DOI: 10.1016/0250-6874(89)87042-8

[6] Hygrometrix Inc. A Comparison of Relative Humidity Sensing Technologies, Applications Note 2004-2 (2004).

[7] J. Domenegueti and S. Zilio. "Humidity and pressure sensor based on internal reflection," Applied Optics 53, 1591-1596 (2014). DOI: 10.1364/AO.53.001591

[8] G. Gruca, D. Chavan, J. Rector, K. Heeck, and D. Iannuzzi. "Demonstration of an optically actuated ferrule-top device for pressure and humidity sensing," Sensors and Actuators A: Physical 190, 77-83 (2013). DOI: 10.1016/j.sna.2012.11.011

[9] F. Pascal-Delannoy, B. Sorli, and A. Boyer. "Quartz crystal microbalance (QCM) used as humidity sensor," Sensors and Actuators A: Physical 84, 285-291 (2000). DOI: 10.1016/S0924-4247(00)00391-5

[10] N. Horzum, D. Tascioglu, C. Ozbek, S. Okur, and M. M. Demir. "VOC sensors based on a metal oxide nanofibrous membrane/QCM system prepared by electrospinning," New Journal of Chemistry 38, 5761-5768 (2014). DOI: 10.1039/c4nj00884g

[11] B. Ding, J. Kim, Y. Miyazaki, and S. Shiratori. "Electrospun nanofibrous membranes coated quartz crystal microbalance as gas sensor for NH3 detection," Sensors and Actuators B: Chemical 101, 373-380 (2004). DOI: 10.1016/j.snb.2004.04.008

[12] J. Hu, F. Zhu, J. Zhang, and H. Gong. "A room temperature indium tin oxide/quartz crystal microbalance gas sensor for nitric oxide," Sensors and Actuators B: Chemical 93, 175-180 (2003). DOI: 10.1016/S0925-4005(03)00186-2

[13] Y. Li, F. Della Valle, M. Simonnet, I. Yamada, and J.-J. Delaunay, "Competitive surface effects of oxygen and water on UV photoresponse of $\mathrm{ZnO}$ nanowires," Applied Physics Letters 94,023110 (2009). DOI: 10.1063/1.3073042

[14] Q. Qi, T. Zhang, Q. Yu, R. Wang, Y. Zeng, L. Liu, and H. Yang. "Properties of humidity sensing $\mathrm{ZnO}$ nanorods-base sensor fabricated by screen-printing," Sensors and Actuators B: Chemical 133, 638-643 (2008). DOI: 10.1016/j.snb.2008.03.035 
[15] G. Zhang, B. E. Patuwo, and M. Y. Hu. "Forecasting with artificial neural networks: The state of the art," International journal of forecasting 14, 35-62 (1998). doi:10.1016/S01692070(97)00044-7

[16] J. F. de Canete, S. Gonzalez-Perez, and P. del Saz-Orozco. "Artificial neural networks for identification and control of a lab-scale distillation column using LabVIEW," World Academy of Science, Engineering and Technology 47, 64-69 (2008).

[17] D.-S. Lee, H.-Y. Jung, J.-W. Lim, M. Lee, S.-W. Ban, J.-S. Huh, and D.-D. Lee. "Explosive gas recognition system using thick film sensor array and neural network," Sensors and Actuators B: Chemical 71, 90-98 (2000). DOI: 10.1016/S0925-4005(00)00614-6

[18] M. Siadat, E. Losson, M. Ghasemi-Varnamkhasti, and S. S. Mohtasebi. "Application of electronic nose to beer recognition using supervised artificial neural networks," in IEEE International Conference on Control, Decision and Information Technologies (CoDIT), (2014) pp. 640-645. DOI: 10.1109/CoDIT.2014.6996971

[19] S. M. Saad, A. M. Andrew, A. Y. M. Shaka_, A. R. M. Saad, A. M. Y. Kamarudin, and A. Zakaria. "Classifying sources influencing indoor air quality (IAQ) using artificial neural network," Sensors 15, 11665-11684 (2015). DOI: 10.3390/s150511665

[20] V. Martinez-Martinez, C. Baladron, J. Gomez-Gil, G. Ruiz-Ruiz, L. M. Navas-Gracia, J. M. Aguiar, and B. Carro. "Temperature and relative humidity estimation and prediction in the tobacco drying process using artificial neural networks," Sensors 12, 14004-14021 (2012). DOI: $10.3390 / \mathrm{s} 121014004$

[21] W. A. Daoud, J. H. Xin, and Y. S. Szeto. "Polyethylenedioxythiophene coatings for humidity, temperature and strain sensing polyamide fibers," Sensors and Actuators B: Chemical 109, 329-333 (2005). DOI: 10.1016/j.snb.2004.12.067

[22] T. Aernouts, P. Vanlaeke, W. Geens, J. Poortmans, P. Heremans, S. Borghs, R. Mertens, R. Andriessen, and L. Leenders. "Printable anodes for flexible organic solar cell modules," Thin solid films 451, 22-25 (2004). DOI: 10.1016/j.tsf.2003.11.038

[23] L. Li, F. Vilela, J. Forgie, P. Skabara, and D. Uttamchandani. "Miniature humidity microsensor based on organic conductive polymer-poly (3, 4-ethylenedioxythiophene)," Micro \& Nano Letters, IET 4, 84-87 (2009). DOI: 10.1049/mnl.2009.0005

[24] J. Liu, M. Agarwal, K. Varahramyan, E. S. Berney, and W. D. Hodo. "Polymer-based microsensor for soil moisture measurement," Sensors and Actuators B: Chemical 129, 599604 (2008). DOI: 10.1016/j.snb.2007.09.017

[25] J.-C. Wang, R. S. Karmakar, Y.-J. Lu, C.-Y. Huang, and K.-C. Wei. "Characterization of piezoresistive PEDOT:PSS pressure sensors with inter-digitated and cross-point electrode structures," Sensors 15, 818-831 (2015). DOI: 10.3390/s150100818

[26] I. W. Kwon, H. J. Son, W. Y. Kim, Y. S. Lee, and H. C. Lee. "Thermistor behavior of PEDOT:PSS thin film," Synthetic Metals 159, 1174-1177 (2009). DOI: 10.1016/j.synthmet.2009.02.006

[27] S. C. Meskers, J. K. van Duren, R. A. Janssen, F. Louwet, and L. Groenendaal. "Infrared detectors with poly (3, 4-ethylenedioxy thiophene)/poly (styrene sulfonic 
acid)(PEDOT/PSS) as the active material," Advanced Materials 15, 613-616 (2003). DOI: $10.1002 /$ adma.200304592

[28] C.-H. Lee, W.-Y. Chuang, S.-H. Lin, W.-J. Wu, and C.-T. Lin. "A printable humidity sensing material based on conductive polymer and nanoparticles composites," Japanese Journal of Applied Physics 52, 05DA08 (2013). DOI: 10.7567/JJAP.52.05DA08

[29] R. Fenner and E. Zdankiewicz. "Micromachined water vapor sensors: a review of sensing technologies," Sensors Journal, IEEE 1, 309-317 (2001). DOI: 10.1109/7361.983470

[30] M. Kuş and S. Okur. "Electrical characterization of PEDOT:PSS beyond humidity saturation," Sensors and Actuators B: Chemical 143, 177-181 (2009). DOI: $10.1016 /$ j.snb.2009.08.055

[31] Sauerbrey, Günter. "Verwendung von Schwingquarzen zur Wägung dünner Schichten und zur Mikrowägung." Zeitschrift für physik 155, no. 2 (1959): 206-222. DOI: $10.1007 / \mathrm{BF} 01337937$

[32] K. K. Kanazawa and J. G. Gordon. "Frequency of a quartz microbalance in contact with liquid," Analytical Chemistry 57, 1770-1771 (1985). DOI: 10.1021/ac00285a062

[33] Muramatsu, Hiroshi, Eiichi Tamiya, and Isao Karube. "Computation of equivalent circuit parameters of quartz crystals in contact with liquids and study of liquid properties." Analytical Chemistry 60, no. 19 (1988): 2142-2146. DOI: $10.1021 / \mathrm{ac} 00170 \mathrm{a} 032$

[34] S. J. Martin, V. E. Granstaff, and G. C. Frye. "Characterization of a quartz crystal microbalance with simultaneous mass and liquid loading," Analytical Chemistry 63, 22722281 (1991). DOI: 10.1021/ac00020a015

[35] S. Brunauer, L. S. Deming, W. E. Deming, and E. Teller. "On a theory of the van der Waals adsorption of gases," Journal of the American Chemical Society 62, 1723-1732 (1940). DOI: $10.1021 / \mathrm{ja} 01864 \mathrm{a} 025$

[36] Nalam, Prathima C., Leonid Daikhin, Rosa M. Espinosa-Marzal, Jarred Clasohm, Michael Urbakh, and Nicholas D. Spencer. "Two-Fluid Model for the Interpretation of Quartz Crystal Microbalance Response: Tuning Properties of Polymer Brushes with Solvent Mixtures." The Journal of Physical Chemistry C117, no. 9 (2013): 4533-4543. DOI: $10.1021 /$ jp310811a

[37] Z. Chen and M.-C. Jin. "An alpha-alumina moisture sensor for relative and absolute humidity measurement," in Industry Applications Society Annual Meeting, 1992., Conference Record of the 1992 IEEE, pp. 1668-1675. DOI: 10.1109/IAS.1992.244236

[38] K. L. Sreenivasan, S. K. Khijwania, T. Philip, and J. P. Singh. "Humidity estimation using neural network and optical fiber sensor," Microwave and Optical Technology Letters 51, 641-645 (2009). DOI: 10.1002/mop.24132 